\author{
Matthias M Müller · Jorge Bosch · Thomas Elbert \\ Andreas Kreiter • Mitchel Valdes Sosa \\ Pedro Valdes Sosa $\cdot$ Brigitte Rockstroh
}

\title{
Visually induced gamma-band responses in human electroencephalographic activity - a link to animal studies
}

\begin{abstract}
Visual presentation of an object produces firing patterns in cell assemblies representing the features of the object. Based on theoretical considerations and animal experiments, it has been suggested that the binding of neuronal representations of the various features is achieved through synchronization of the oscillatory firing patterns. The present study demonstrates that stimulus-induced gamma-band responses can be recorded noninvasively from human subjects attending to a single moving bar. This finding indicates the synchronization of oscillatory activity in a large group of cortical neurons. Gamma-band responses were not as apparent in the presence of two independently moving stimuli, suggesting that the neuronal activity patterns of different objects are not synchronized. These results open a new paradigm for investigating the mechanisms of feature binding and association building in relation to subjective perception.
\end{abstract}

Key words Visually-induced gamma-band responses . Coherency $\cdot$ Evolutionary spectra $\cdot$ Human

\section{Introduction}

It is generally accepted that perceived objects are represented by a pattern of graded activity in neuronal cell assemblies rather than by an isolated response of a single cell (e.g., a "grandmother cell"). When multiple stimuli are processed in parallel, a functional separation of the spatially overlapping cell assemblies that pertain to the different stimuli is necessary in order to avoid false con-

M.M. Müller ( T. Elbert · B. Rockstroh

Department of Psychology, University of Konstanz,

P.O. Box 5560, D-78434 Konstanz, Germany;

e-mail: matthias.m.mueller@uni-konstanz.de

J. Bosch · M.V. Sosa P. P. Sosa

Cuban Center of Neuroscience, Ave. 25, Cubanacan,

La Havana, Cuba

A. Kreiter

Max-Planck-Institute for Brain Research, Deutschordenstrasse 46 , D-60528 Frankfurt am Main, Germany junctions. Theoretical considerations and experimental results suggest that synchronization of neuronal responses participating in the representation of the same stimulus would be a particularly useful mechanism to dynamically bind related responses for further joint processing (Milner 1974; von der Malsburg and Schneider 1986; Singer and Gray 1995). Furthermore, intracortical recordings in animals suggest that synchronized activity is often associated with oscillatory patterns of activity in the gamma range (above 25-30 Hz; Gray et al. 1989; Eckhorn et al. 1990; Engel et al. 1991a-c, 1992, Kreiter and Singer 1992; König et al. 1995). These phenomena are called induced gamma-band oscillations. Moving bars have been used as stimuli to induce gamma-band responses in the visual cortex of anesthetized cats (e.g., Eckhorn et al. 1990; Engel et al. 1991a-c), and awake behaving monkeys (Kreiter and Singer 1992), in the optic tectum of pigeons (Neuenschwander and Varela 1993), and in the dorsal cortex and the dorsal ventricular ridge of pond turtles (Prechtl 1994). However, some studies in monkeys have failed to detect oscillations when using stimuli such as moving light bars (Young et al. 1992), dynamic random-dot displays with a fraction of dots moving coherently (Bair et al. 1994), and static stimuli (Tovee and Rolls 1992). It may be the case that recordings at the cellular level may not reflect the synchronization of large populations of neurons. Rhythmic activity that is prominent at the level of field potentials, may not be apparent at the level of single-unit activity (Young et al. 1992; Singer and Gray 1995). The question arises whether or not it is possible to detect oscillations in cell assemblies using macroscopic recordings such as electrocorticographic $(\mathrm{ECOG})$ or electroencephalographic activity (EEG). If so, the relation between gamma-band responses and perceptual processes could be investigated in humans.

In contrast to intracranial recordings in animal experiments, noninvasive investigations of induced gammaband responses in humans is fraught with problems. The first consideration should concern methodological issues. In contrast to previously reported evoked gamma-band responses in the auditory and visual modality (Makeig 
1993; Galambos 1992; Pantev et al. 1993; Tiitinen et al. 1993), induced gamma-band responses cannot be extracted by conventional averaging, since the oscillatory spindles are not phase-locked to the stimulus (Gray et al. 1989; Eckhorn et al. 1990, 1993; Engel et al. 1991a, b, 1992; Kreiter and Singer 1992; Makeig 1993; Bauer et al. 1995; König et al. 1995). In addition, the duration of synchronous firing is brief (100-200 ms in cats; Gray et al. 1989). Indications of induced gamma-band responses in humans had been previously reported for visual (Lutzenberger et al. 1995), auditory (Jokeit and Makeig 1994), and sensorimotor (Kristeva-Feige et al. 1993) stimulation and during word processing (Pulvermüller et al. 1994a, b). In these studies power spectra were calculated by using fast fourier transformations (FFT) with short and overlapping data windows. It is, however, artificial and suboptimal to decompose a brief and transient signal using a series of infinitely extended basis functions. Instead of composing the signals by means of an inadequate series of sine and cosine functions and then restricting the arising problems by multiplying the time series with a window function (Fourier methods), Gabor (1946) suggested to directly use functions of finite length. The Gabor transformation decomposes the time series into complex exponentials modulated by fixed-length Gaussian windows. A finite signal is better represented by a sum of finite (e.g., Gabor) functions. Therefore, we used Gabor transforms for the spectral analyses in the present study.

A second consideration involves anatomical problems. Most parts of area 17 (V1) in humans are located in the gyrus occitemporalis medialis of the respective hemifield. Visual fullfield stimulation produces opposing electrical dipoles causing cancellation of their electrical signal. Therefore visual stimuli were presented to one hemifield.

The current investigation was designed to show that coherent oscillatory activity patterns, i.e., induced gamma-band responses can be recorded noninvasively from the human brain, similar to the response patterns found in animals. It also investigated these activity patterns in respect to global properties of the stimulus.

\section{Materials and methods}

The experimental design was modeled after previous animal studies (Gray et al. 1989). There were two stimulus configurations: the first (coherent) consisted of a single moving bar, which was expected to activate a single, synchronously firing neuronal assembly; the second (incoherent) contained two identical bars, moving in opposite directions (see Fig. 2, inset). The latter stimulus was expected to activate separate neuronal assemblies, thus producing desynchronous responses. Bars were presented in the left visual hemifield in order to avoid cancellation of superimposed electrical vectors of cortical activity due to folding within the visual cortex.

Dimensions of the light bars were $9.8^{\circ} \times 0.46^{\circ}$ for the coherent and $4^{\circ} \times 0.46^{\circ}$ for the incoherent stimulus condition. For the coherent-motion condition the bar started $9.64^{\circ}$ away from the fixation point and moved toward the center of the screen at a velocity of $1.9^{\circ} \mathrm{s}$ (see inset, Fig. 2A). This characterized also the motion of one of the two bars for the incoherent-motion condition; the second bar started at $2.58^{\circ}$ (inset Fig. 2B). Luminance of bars and background was 1.0 and $0.05 \mathrm{~cd} / \mathrm{m}^{2}$, respectively. Each condition included 100 stimuli. Coherent and incoherent conditions were presented in a random order with an interstimulus interval of 1750 $\mathrm{ms}$. Prior to motion onset, bars were presented as standing stimuli for $260 \mathrm{~ms}$ and then stayed in motion for $3700 \mathrm{~ms}$. Stimuli were presented on a 20 -inch monitor with a frame rate of $60 \mathrm{~Hz}$ (noninterlaced) positioned $1 \mathrm{~m}$ in front of the subject. Subjects were instructed to fixate on a cross at the center of a screen while the stimuli were presented. Eight subjects were paid for participation (U.S. \$ 15). All had normal vision and were naive with respect to the scientific goals of the study. The EEG (pass band $0.3-300 \mathrm{~Hz}$ ) was recorded using $\mathrm{Ag}-\mathrm{AgCl}$ electrodes from $\mathrm{Pz}, \mathrm{Oz}, \mathrm{P} 3, \mathrm{P} 4, \mathrm{~T}$, $\mathrm{T} 6, \mathrm{O} 1, \mathrm{O} 2, \mathrm{VEOG}$ referenced to linked earlobes (international $10-20$ system). Electrode impedance was below $5 \mathrm{k} \Omega$. The sampling rate was set to $1000 \mathrm{~Hz}$. Trials with electro-oculographic (EOG) artifacts were rejected when the absolute value of the amplitude exceeded $75 \mu \mathrm{V}$. One subject, whose data contained more than $60 \%$ artifacts, was removed from further analysis. Thus seven subjects (mean age 26.1 years; four men, three women) remained for the analysis. The mean number of trials that remained per subject for the coherent-motion was 96 and 94 for the incoherent-motion condition.

Frequency analysis

The evolutionary spectrum (Priestley 1988) was used to determine time changes in the intensity of oscillatory activity. It is specifically assumed that the $i$ th recording epoch can be modeled by:

$V_{i}(t)=\Pi(t)+\beta_{i}(t)$

where $\Pi(t)$ is the mean evoked response (or the event-related potential) and $\beta_{i}(t)$ the trial-by-trial fluctuation around the mean. Since the present analyses focused on oscillatory activity which is not deterministically time or phase-locked to the stimuli, the usual estimate of $\Pi(t)$ was subtracted from each epoch. An estimate of the evolutionary spectrum of $\beta_{i}(t)$ was calculated by means of the discrete Gabor transform (Qian and Chen 1993). A time and frequency resolution compatible with the time/frequency uncertainty principle must be selected. In our case this was $64 \mathrm{~ms}$ for time and $3.9 \mathrm{~Hz}$ for frequency (from 0.48 to $125 \mathrm{~Hz}$, with downsampling for analysis for $250 \mathrm{~Hz}$ ). This was achieved by sliding a time window, $256 \mathrm{~ms}$ in length, in 64-ms steps across the time series. Then a single transformation is obtained which is equivalent to the following steps:

1. A sliding window $\gamma(t)$ is used to select segments of data centered around each time $t$ and down weighted toward the extremes. $\gamma(t)$ is calculated as described by Quin and Chen (1993) such that it has minimum energy, most closely approximates a Gaussian function and ensures minimal crosstalk between adjacent frequencies.

2. The spectrum is computed for each segment. A combination of steps 1 and 2 yields the discrete Gabor transform coefficients $G_{i}(t, \omega)$, where $\omega$ is the frequency. It must be noted that in this way an invertible Discrete Gabor transform is ensured. Finally, the variance of the complex random variates $G_{i}(t, \omega)$ over all epochs $i$ is computed in order to form a consistent estimate of the evolutionary spectrum. In population terms:

$\sigma_{\beta}^{2}(t, \omega)=E\left[\left|G_{i}(t, \omega)\right|^{2}\right]$

where $\sigma_{\beta}^{2}(t, \omega)$ is the evolutionary spectrum of the $\beta_{i}(t), \mathbf{E}[x]$ denotes the expected value operator of a random variable $x$ and $|a|^{2}$ denotes the absolute value of a complex number $a$.

\section{Macaque monkey}

The ability of this analysis method to extract induced gamma-band responses typically observed in animal studies was tested in a first step for local field potential recordings from area 17 of an awake, fixating macaque monkey (Macaca fascicularis). The stimulus was a $1^{\circ} \times 1^{\circ}$ light bar moving with a velocity of $1 \%$ over the receptive field. For intracortical extracellular recording, glass-insulated el- 

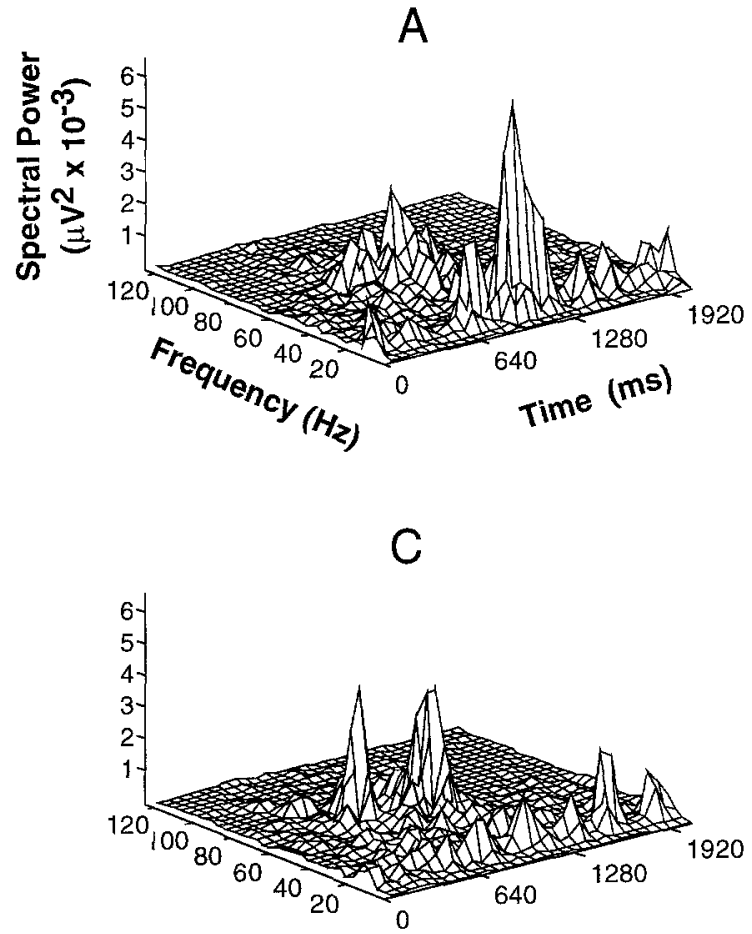

Fig. 1 Detection of induced gamma responses in the local-field potentials recorded in $\mathrm{V} 1$ of an awake fixating monkey (Macaca fascicularis) A-C Examples of responses extracted from single sweeps. The mean of ten single sweeps is presented in the lower right quadrant. $x$-axis, time in milliseconds. $y$-axis, frequency $4-120 \mathrm{~Hz}$. $z$-axis, square of the absolute spectral value (microvolts squared $\times 10^{-3}$ )

giloy electrodes $(1 \mathrm{M} \Omega$ at $100 \mathrm{~Hz}$ ) have been used. Data were recorded with a sampling frequency of $1000 \mathrm{~Hz}$ and the raw signal was filtered from 10 to $100 \mathrm{~Hz}$. A total of ten trials was recorded.

All surgical and behavioral procedures were performed in accordance with the guidelines for the welfare of experimental animals issued by the Federal Government of Germany. The training of the animal, the implantation of the recording chamber, and the description of the recording session was extensively reported by Kreiter (1992) and Kreiter and Singer (1992).

\section{Data reduction and statistical analysis for human EEG data}

Signal power was statistically analyzed in two frequency bands: a broad gamma band ( $40-96 \mathrm{~Hz})$ and the alpha band $(8-12 \mathrm{~Hz})$. The standing stimuli interval (i.e., the first $260 \mathrm{~ms}$ ) served as a baseline. ${ }^{1}$ The mean spectral power for each frequency and electrode was calculated for every subject, on the basis of the single-trial evolutionary spectra. The first $1400 \mathrm{~ms}$ while stimuli were in motion were divided into seven time windows (192 ms each, no overlap). Given that the time resolution of the evolutionary spectra was $64 \mathrm{~ms}$, the time windows chosen represent the summation of three successive time points of the evolutionary spectra. Scores for alpha and gamma were obtained by averaging across the respective frequencies. Each of the seven time windows was normalized by dividing it by the mean spectral power of the baseline (standing stimuli) for each electrode. In order to approximate a Gaussian distribution, natural logarithms were calculated for all normalized data points. To test the normal-

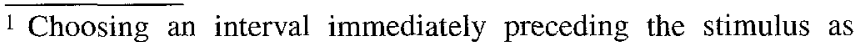
baseline interval would not have affected any of the reported differences between the coherent-motion and the incoherent-motion conditions.
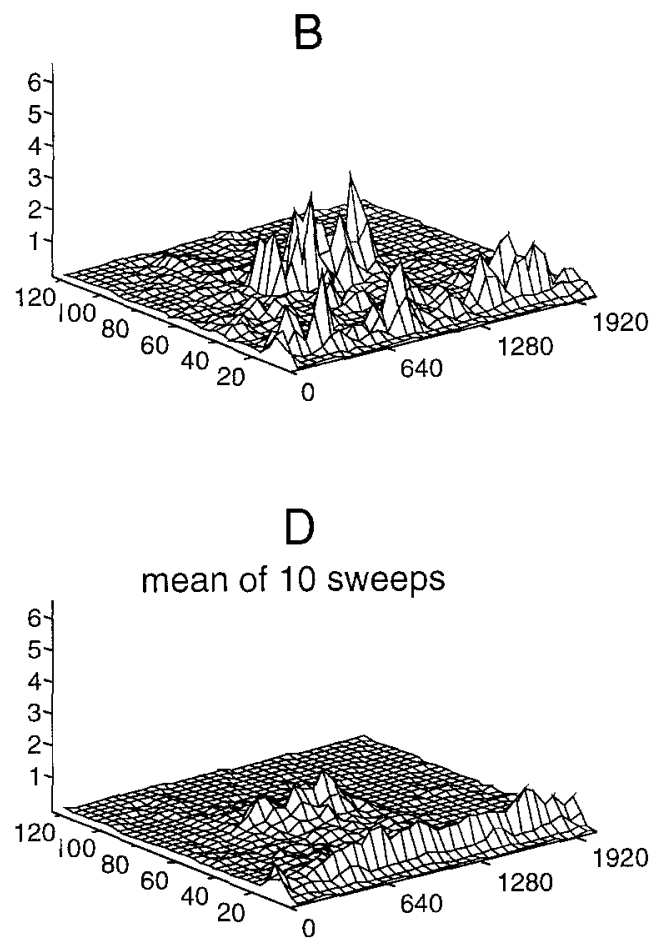

ized mean over all time windows for coherent and incoherent motion, mean comparisons were calculated over the summed logarithms. $P$-values were adjusted by Huynh-Feldt correction. $t$-Tests for each time window were used to inspect the normalized power values for the coherent and incoherent conditions.

\section{Results}

Local field potentials in the macaque monkey

Figure 1 depicts three examples of an evolutionary spectrum from the monkey local-field recordings. Examples of single sweeps and the grand mean of evolutionary spectra of ten successive single sweeps is illustrated. Spectral power was centered around $60 \mathrm{~Hz}$. Figure $1 \mathrm{~A}-\mathrm{C}$ demonstrate the variance of frequency and time in the onset of the induced gamma-band activity across the single sweeps. These findings correspond to those typically described in animal studies and parallel the observations of Kreiter (1992). Averaging of evolutionary spectra across all ten single sweeps resulted in a reduction and a widening of spectral power (Fig. 1D).

\section{Human EEG}

Grand mean normalized evolutionary spectra for the human subjects are shown in Fig. 2. Figure 2A represents coherent and Fig. 2B incoherent motion. Relative to the standing stimuli, the grand means of the evolutionary spectra for the different electrode positions revealed several important findings:

1. the single-bar condition (Fig. 2A) was associated with enhanced gamma-band $(40-96 \mathrm{~Hz})$ activity.

2. This activity was most pronounced over the contralateral parieto-occipital scalp sites $(\mathrm{P} 4, \mathrm{~T} 6, \mathrm{O} 2)$ and ap- 

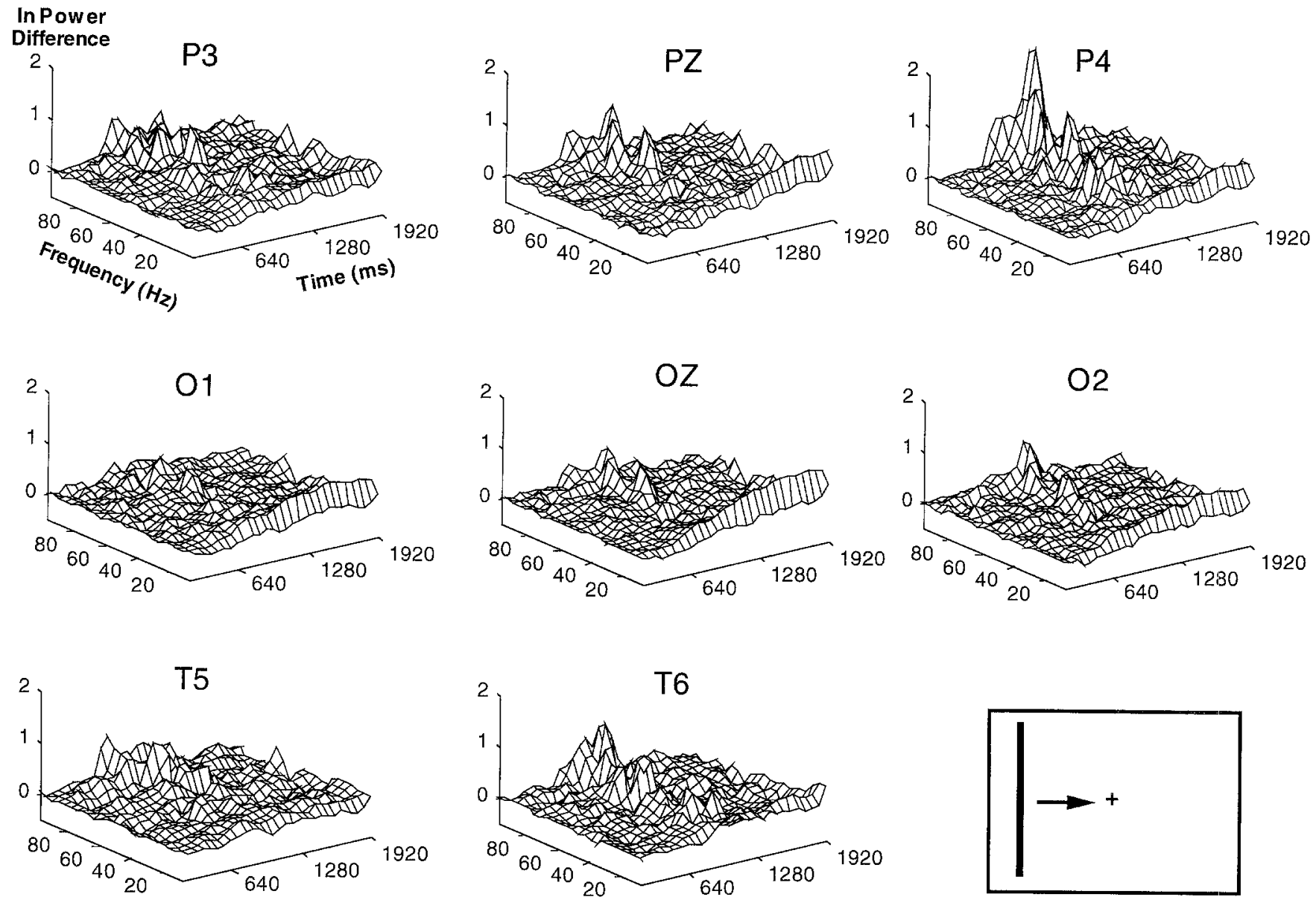

\section{$2 \mathrm{~A}$}

Fig. 2 Normalized grand mean of the time-resolved spectra over all subjects for the coherent (A) and the incoherent moving-bar stimulus (B). $x$-axis, time in milliseconds. The standard stimuli time period from 0 to $260 \mathrm{~ms}$ served as baseline. $y$-axis, frequency 4-96 Hz. z-axis, difference in ln spectral power: response during motion minus baseline (standing stimulus)

peared in a time window between about 400 and $900 \mathrm{~ms}$ after motion onset. For the electrode P4 it exceeded the level that was present during the standing stimulus by an order of magnitude (note that log-values are plotted on the z-axis in Fig. 2).

3. In the presence of two incoherently moving bars, no such enhancement of activity in the gamma range was observed (Figs. 2B, 3A).

4. Alpha activity was less pronounced in coherent as compared to incoherent motion.

5. Alpha blocking was equally prominent during the standing stimuli and at the beginning of the motion. The effect of alpha blocking dissipated during the time course of motion. Figure $3 \mathrm{~B}$ illustrates the change of alpha activity referred to the value for the standing stimulus, i.e., to a time when alpha activity was blocked. The subsequent augmentation, apparent in Fig. 3B, therefore, indicates recovery back to intertrial levels.
6. Topography and time course of alpha activity are different from the patterns in any other frequency band. This was true for both experimental conditions.

Statistical analysis of the normalized time windows showed significantly larger spectral power in the gamma band in all time windows for coherent motion as compared to incoherent motion $\left(F_{(1,65)}=9.34, P<0.05\right)$. Differences were most pronounced for the time window $448-640 \mathrm{~ms}$ $(t=2.0, P=0.05)$ and $640-832 \mathrm{~ms}$ after motion onset $(t=2.5$, $P=0.01$; Fig. $3 \mathrm{~A}$ ). In contrast, the relative changes in power over all time windows in the alpha band $(8-12 \mathrm{~Hz})$ was significantly lower in coherent as compared to incoherent motion $\left(F_{(1,65)}=10.21, P<0.01\right.$; Fig. 3B $)$.

\section{Discussion}

When the Gabor transform was applied to the intracranial recordings of local field potentials from area 17 of an awake behaving monkey, the synchronized oscillating spindles in the gamma band were readily extracted. The evolutionary spectra obtained from single sweeps are consistent with the results obtained in previous animal investigations: cell groups in area 17 exhibited in-phase firing if they were activated by a coherent stimulus (e.g., long bar) even if their receptive fields were not overlapping. If two bars moving in 

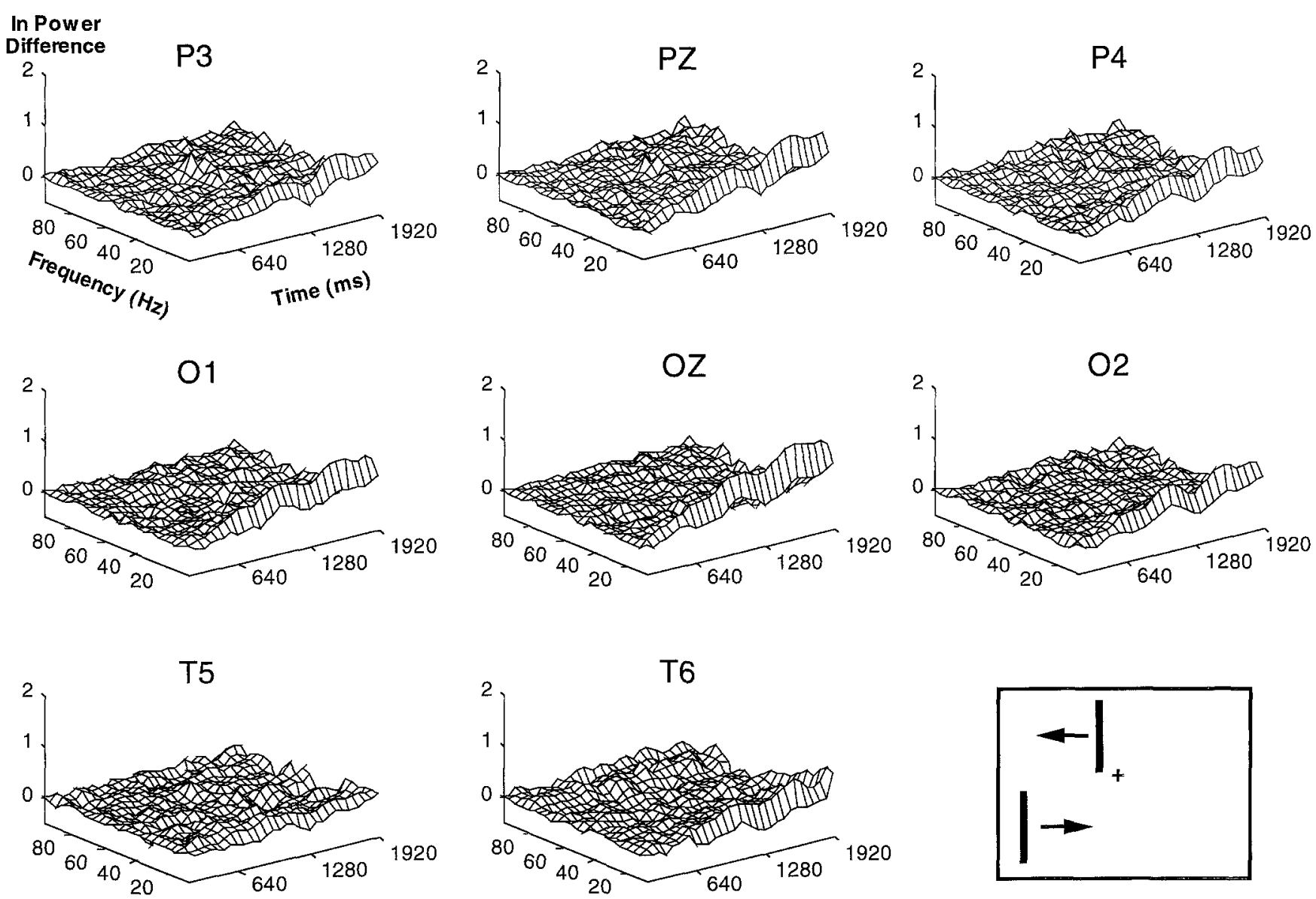

\section{B}

opposite directions were presented, firing remained uncorrelated (Gray et al. 1989; Engel et al. 1992).

Averaging the presently obtained spectra across ten single sweeps resulted in a reduced signal power as the time of onset and the frequency of the spindles varied from trial to trial. Despite the fact that the signal is reduced, the averaging process should still enhance the signal-to-noise ratio. This is of particular importance in cases where the biological noise is large, as in human EEG.

The present results correspondingly show that a coherent moving-bar stimulus induces gamma-band responses in the human EEG. When the stimuli were independently moving bars, such a response was not produced. This observation can be taken as evidence for synchronized activity in extended neuronal networks in response to the coherent condition. The lack of an equally strong gammaband response for the incoherent condition may be explained by assuming that two or more neuronal assemblies are firing independently. On the average, out-of-phase signals tend to cancel each other in EEG recordings.

In cat visual cortex, synchronization had been demonstrated not only within but also across individual areas, e.g., between areas 17, 18, and 19 (Eckhorn et al. 1988) or between area 17 and PLMS (posteromedial lateral suprasylvian area) (Engel et al. 1991c). The topography of the presently obtained human EEG responses suggests that higher-order visual areas contributed to the measured activity. Induced gamma-band responses to the coherent stimulus were most pronounced at electrode sites P4 and T6. Responses at these sites greatly exceeded the baseline activity. It is possible that synchronized oscillations are produced by cell assemblies in the middle temporal visual area (Kreiter and Singer 1992) or V5, an area which is thought to be involved in motion processing in humans. The location of human V5, as suggested by positron emission tomography studies (Zeki 1993), would be consistent with the observed EEG distribution.

Recently Jürgens and coworkers (1995) argued that gamma-band responses may be nothing but harmonics within the alpha band. In the present study, alpha and gamma activity displayed a different behavior in both time course and topography. This can be derived from the examination of the evolutionary spectra. During coherent stimulus presentation, enhanced activity in the gamma band covaried with reduced alpha activity. These observations lead to the conclusion that the observed responses in the gamma band cannot be considered harmonics of alpha-waves.

In contrast to intracranial recordings, surface EEG recordings may be contaminated by electromyographic (EMG) activity. Neither the maximum of the gammaband activity on the scalp contralateral to the stimulated 
In Power Difference

(Response - Baseline)

\section{Gamma-band 40-96 Hz left visual hemi-field}

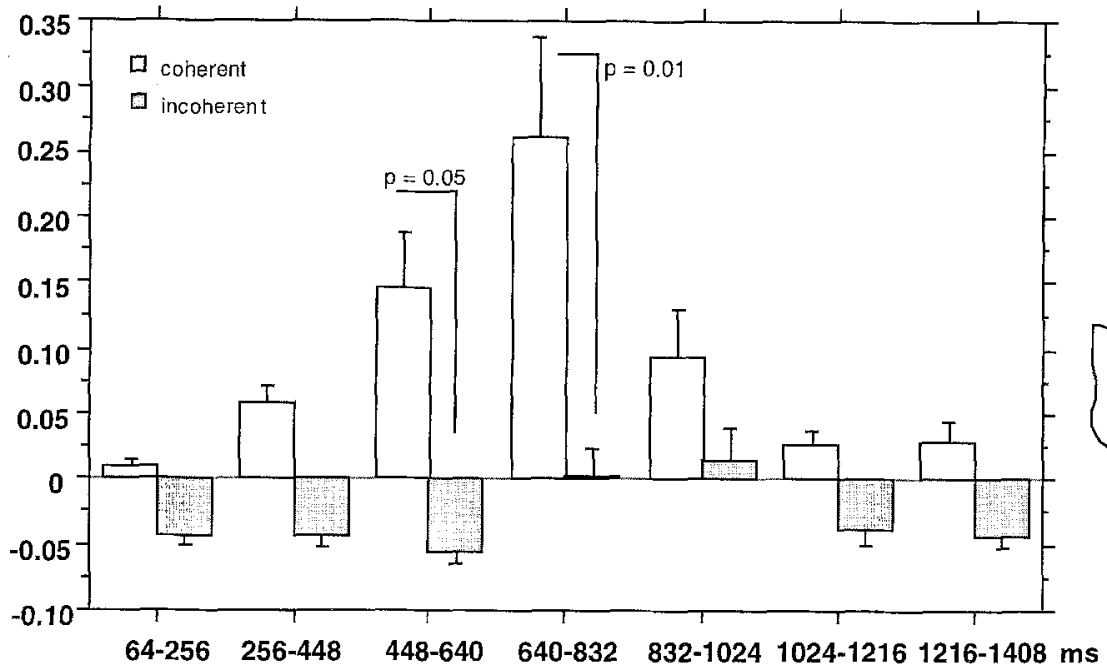

A

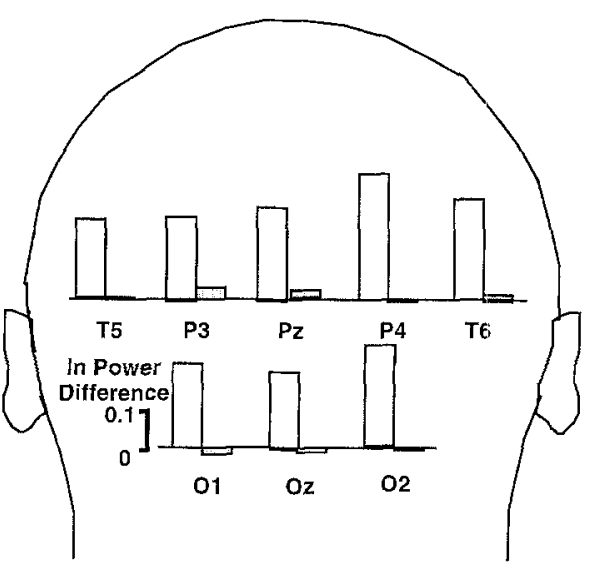

Amplitudes time window $640-832 \mathrm{~ms}$ for coherent (left white bar) and incoherent (right shadedbar) movement for each electrode

In Power Difference

(Response - Baseline)

Alpha-band 8-12 Hz left visual hemi-field
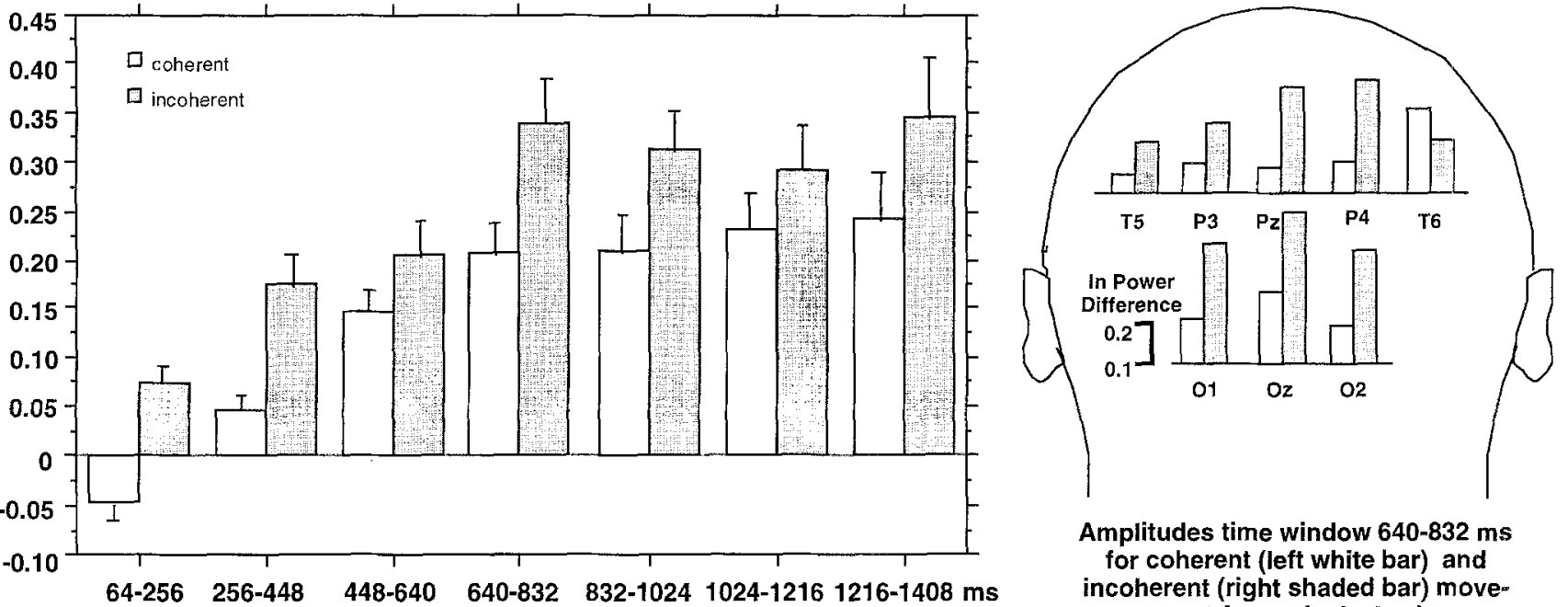

Amplitudes time window $640-832 \mathrm{~ms}$ for coherent (left white bar) and incoherent (right shaded bar) movement for each electrode

B

Fig. 3 Means and SEs averaged across all electrodes and subjects for the logarithmis power of normalized spectral gamma band (A) and alpha band (B) referred to. The seven 192-ms nonoverlapping windows for coherent and incoherent motion are presented separately. Right, the mean amplitudes for coherent (left white bars) and incoherent stimulus conditions (right shaded ars) are presented for the time window 640-832 ms separately for each electrode. In $\mathbf{B}$ (right), the offset of the $x$-axis is 0.1

hemifield nor the dependence of the response on the stimulus conditions are a likely effect of muscular activity. Furthermore, EMG spectra typically have a different characteristic (Cacioppo 1990), whereby no such distinct peaks occur, as seen in the current results.

In cat visual cortex the reported latencies for the first oscillatory spindles not phase-locked to the moving stimuli were around $400 \mathrm{~ms}$ (Eckhorn et al. 1990; Bauer et al. 1995). This is consistent with the present findings, where the first activity elicited by coherent motion does not appear earlier than the 256-448 ms time bin (Fig. 3). However, stimulus-evoked activity (i.e., responses phase-locked to the stimulus) occur within the first $100 \mathrm{~ms}$ after stimulus presentation (Eckhorn et al. 1990; Pantev et al. 1994; Bauer et al. 1995). In the present study stimulus-evoked activity, the evoked potential, was subtracted prior to the calculation of the evolutionary spectra. The late appearance of stimulus-induced activity suggests a possible fundamental difference between evoked and induced oscillations. Eckhorn and coworkers (1990) speculated on the complementary features of the two types of oscillations. They assumed that "evoked responses serve to define crude instantaneous 
'preattentive percepts,' and stimulus-induced oscillatory synchronization mainly support the formation of more complex, 'attentive percepts' that require iterative interactions among different processing levels and memory" (p. 302). The latency of the induced gamma-band responses would be in agreement with this interpretation.

The functional relevance of induced gamma-band responses is still in debate (e.g., Tovee and Rolls 1992; Young et al. 1992; Bair et al. 1994; Singer and Gray 1995). Evidence that induced gamma-band responses are possibly linked to feature binding was recently put forth by Tallon and coworkers (1995). The presentation of an illusory triangle (Kanizsa triangle) produced $30-\mathrm{Hz}$ activity most pronounced around the vertex $(\mathrm{Cz})$. A control stimulus without the illusory quality (achieved by rotation of the induced disks) elicited no such gamma-band response. This finding, together with the present results, lends strong support to the hypothesis that macroscopically visible gamma-band activity is functionally relevant to feature binding.

Acknowledgements We are grateful to Antonieta Maria Bobes for help in recording the data of the human subjects. We thank Drs. Ted Bullock, Steven Hillyard, Scott Makeig, and an anonymous reviewer for their constructive comments on the manuscript and Dorothy Charbonnier, $\mathrm{PhD}$, for her editorial assistance. This work was supported by grants from the Deutsche Forschungsgemeinschaft and the Human Frontier Science Program (HFSP, RG 20/95).

\section{References}

Bair W, Koch C, Newsome W, Britten K (1994) Power spectrum analysis of bursting cells in area MT in the behaving monkey. J Neurosci 14:2870-2892

Bauer R, Brosch M, Eckhorn R (1995) Different rules of spatial summation from beyond the receptive field for spike rates and oscillation amplitudes in cat visual cortex. Brain Res 669: 291-297

Cacioppo JT (1990) The skelemotor system. In: Cacioppo JT, Tassinary LG (eds) Principles of psychophysiology. Cambridge University Press, Cambridge, pp 325-384

Eckhorn R, Bauer R, Jordan W, Brosch M, Kruse W, Munk M, Reitboeck HJ (1988) Coherent oscillations: a mechanism of feature linking in the visual cortex. Biol Cybern 60:121-130

Eckhorn R, Frien A, Bauer R, Woelbern T, Kehr H (1993) High frequency $(60-90 \mathrm{~Hz})$ oscillations in primary visual cortex of awake monkey. Neuroreport 4:243-246

Eckhorn R, Reitboeck HJ, Arndt M, Dicke P (1990) Feature linking via synchronization among distributed assemblies: simulations of results from cat visual cortex. Neural Computation 2: 293-307

Engel AK, König P, Kreiter AK, Gray CM, Singer W (1991a) Temporal coding by coherent oscillations as a potential solution to the binding problem: physiological evidence. In: Schuster HG (ed) Nonlinear dynamics and neuronal networks. VHC, Weinheim, pp 3-25

Engel AK., König P, Kreiter AK, Singer W (1991b) Interhemispheric synchronization of oszillatory neuronal responses in cat visual cortex. Science 252:1177-1179

Engel AK, Kreiter AK, König P, Singer W (1991c) Synchronization of oscillatory neuronal responses between striate and extrastriate visual cortical areas of the cat. PNAS 88:9136-9140

Engel AK, König P, Kreiter AK, Schillen TB, Singer W (1992) Temporal coding in the visual cortex: new vistas on integration in the nervous system. Trends Neurosci 15:218-226

Gabor D (1946) Theory of communications. Proc IEE 93:429-457

Galambos R (1992) A comparison of certain gamma band $(40-\mathrm{Hz})$ brain rhythms in cat and man. In: Basar E, Bullock TH (ed) Induced rhythms in the brain. Birkhäuser, Boston, pp 201-216
Gray CM, König P, Engel AK, Singer W (1989) Oscillatory responses in cat visual cortex exhibit inter-columnar synchronization which reflects global stimulus properties. Nature 338:334-337

Jokeit H, Makeig S (1994) Different event-related patterns of gamma-band power in brain waves of fast- and slow-reacting subjects. PNAS 91:6339-6343

Jürgens E, Rösler F, Henninghausen E, Heil M (1995) Stimulus-induced gamma oscillations: harmonics of alpha activity? Neuroreport 6:813-816

König P, Engel AK, Singer W (1995) Relation between oscillatory activity and long-range synchronization in cat visual cortex. PNAS 92:290-294

Kreiter AK (1992) Kodierung neuronaler Assemblies durch kohärente Aktivität: Korrelationsanalysen im Sehsystem von Säugetieren. Eberhard-Karls-Universität, Tübingen ( $\mathrm{PhD}$ thesis)

Kreiter AK, Singer W (1992) Oscillatory neuronal responses in the visual cortex of the awake macaque monkey. Europ J Neurosci 4:369-375

Kreiter AK, Singer W (1994) Global stimulus arrangement determines synchronization of neuronal activity in the awake macaca monkey. Eur J Neurosci [Suppl] 7:153

Kristeva-Feige R, Feige B, Makeig S, Ross B, Elbert T (1993) Oscillatory brain activity during a motor task. Neuroreport 4: 1291-1294

Lutzenberger W, Pulvermüller F, Elbert T, Birbaumer N (1995) Visual stimulation alters local $40-\mathrm{Hz}$ responses in humans: an EEG-study. Neurosci Lett 183:39-42

Makeig S (1993) Auditory event-related dynamics of the EEG spectrum and effects of exposure to tones. Electroencephalogr Clin Neurophysiol 86:283-293

Milner PM (1974) A model for visual shape recognition. Psychol Rev 81:521-535

Neuenschwander S, Varela FJ (1993) Visually triggered neuronal oscillations in the pigeon: an autocorrelation study of tectal activity. Eur J Neurosci 5:870-881

Pantev C, Elbert T, Makeig S, Hampson S, Eulitz C, Hoke M (1993) Relationship of transient and steady-state auditory evoked fields. Electroencephalogr Clin Neurophysiol 88:389-396

Pantev C, Elbert T, Lütkenhöner B (eds) (1994) Oscillatory eventrelated brain dyamics. Plenum, New York

Pulvermüller F, Preissl H, Eulitz C, Pantev C, Lutzenberger W, Elbert T, Birbaumer N (1994a) Brain rhythms, cell assemblies and cognition: evidence from the processing of words and pseudowords. Psycoloquy 5:48

Pulvermüller F, Preissl H, Eulitz C, Pantev C, Lutzenberger W, Elbert T, Birbaumer N (1994b) Gamma-band responses reflect word/pseudoword processing. In: Pantev C, Elbert T, Lütkenhöner B (eds) Oscillatory event-related brain dymanics. Plenum, New York

Prechtl JC (1994) Visual motion induces synchronous oscillations in turtle visual cortex. PNAS 91:12467-12471

Priestley MB (1988) Non-linear and non-stationary time series analysis. Academic, London

Qian S, Chen D (1993) Discrete Gabor transform. IEEE Trans Signal Proc 41:2429-2438

Singer W, Gray CM (1995) Visual feature integration and the temporal correlation hypothesis. Annu Rev Neurosci 18:555-586

Tallon C, Bertrand O, Bouchet P, Pernier J (1995) Gamma-range activity evoked by coherent visual stimuli in humans. Eur J Neursci 7:1285-1291

Tiitinen H, Sinkkonen J, Reinikainen K, Alho K, Lavikainen J, Näätänen R (1993) Selective attention enhances the auditory $40-\mathrm{Hz}$ transient response in humans. Nature 364:59-60

Tovee MJ, Rolls ET (1992) Oscillatory activity is not evident in the primate temporal visual cortex with static stimuli. Neuroreport 3:369-372

Malsburg C von der, Schneider W (1986) A neural cocktail-party processor. Biol Cybern 54:29-40

Young MP, Tanaka K, Yamane S (1992) On oscillating neuronal responses in the visual cortex of the monkey. $J$ Neurophysiol $67: 1464-1474$

Zeki S (1993) A vision of the brain. Blackwell Scientific, London 